\title{
Overexpression of GSTP1 promotes colorectal cancer cell proliferation, invasion and metastasis by upregulating STAT3
}

\author{
Feifei Wang ${ }^{1,2, B-D, F}$, Ceng Zhang ${ }^{1,2, B, C, F}$, Xiaohui Zhu ${ }^{1,2, B, C, F}$, Dan Zhang ${ }^{1,2, B, F}$, Zhaowen Zhang ${ }^{1,2, C, F}$, \\ Shunjie $\mathrm{Ni}^{3,, C, F}$, Zhizhi Wang ${ }^{4, C, F}$, Shuyi X ${ }^{5, C, F}$, Xiaoliang Lan ${ }^{6, C, F}$, Yanqing Ding ${ }^{1,2, C, F}$, Li Liang ${ }^{1,2, A, E, F}$ \\ ${ }^{1}$ Department of Pathology, Nanfang Hospital, Southern Medical University, Guangzhou, China \\ ${ }^{2}$ Guangdong Province Key Laboratory of Molecular Tumor Pathology, Basic Medical College, Southern Medical University, Guangzhou, China \\ ${ }^{3}$ Department of Neurology, Nanfang Hospital, Southern Medical University, Guangzhou, China \\ ${ }^{4}$ Department of Thoracic Surgery, Nanfang Hospital, Southern Medical University, Guangzhou, China \\ ${ }^{5}$ Basic Medical College, Southern Medical University, Guangzhou, China \\ ${ }^{6}$ Department of General Surgery, Nanfang Hospital, Southern Medical University, Guangzhou, China \\ A - research concept and design; $B$ - collection and/or assembly of data; $C$ - data analysis and interpretation; \\ $D$ - writing the article; $E$ - critical revision of the article; $F$ - final approval of the article
}

Address for correspondence

Li Liang

E-mail: lli@fimmu.com

Funding sources

The National Key R\&D Program of China (grant

No. 2017YF(1309002), the National Natural Science Foundation

of China (grants No. 81672821, No. 81872041, №. 81802306, and No. 81903002), China Postdoctoral Science Foundation (grants No. 2018M643125 and No. 2019M652963), the Natural Science Foundation Key Program of Guangdong Province of China (grant No. 2018B0303110017), and the Natural Science Foundation of Guangdong Province of China (grant No. 2019A1515011436)

Conflict of interest

None declared

Received on May 10, 2021

Reviewed on August 7, 2021

Accepted on September 20, 2021

Published online on February 23, 2022

Cite as

Wang F, Zhang C, Zhu X, et al. Overexpression of GSTP7 promotes colorectal cancer cell proliferation, invasion and metastasis by upregulating STAT3. Adv Clin Exp Med. 2022;31(2):139-149

doi:10.17219/acem/142461

DOI

10.17219/acem/142461

Copyright

Copyright by Author(s)

This is an article distributed under the terms of the

Creative Commons Attribution 3.0 Unported (CC BY 3.0)

(https://creativecommons.org/licenses/by/3.0/)

\section{Abstract}

Background. The abnormal expression of glutathione S-transferase P1 (GSTP1) is associated with the progression of several tumor types. However, its role and molecular mechanism in the progression of colorectal cancer (CRC) are largely unknown.

Objectives. To examine the effect of GSTP1 in CRC and determine its possible mechanisms.

Materials and methods. In the present study, immunohistochemistry (IHC) and the quantitative reverse transcription polymerase chain reaction (qRT-PCR) analysis were used to detect the expression of GSTP1 and signal transducer and activator of transcription 3 (STAT3) in CRC tissues. Western blotting was applied to detect the expression of GSTP1 and proteins of the Janus kinase (JAK)-STAT3 pathway in different CRC cell lines. The interaction and co-localization of GSTP1 and STAT3 were detected using co-immunoprecipitation (co-IP) and immunofluorescence (IF) in the SW620 cell line.

Results. A positive correlation was identified between the expression of GSTP1 and STAT3 in human CRC tissues. The overexpression of GSTP1 promoted the proliferation, invasion and metastasis of CRC cells by upregulating STAT3. The GSTP1 and STAT3 can directly bind to and regulate each other. The interaction between them is regulated by the upstream gene called F-box only protein 8 (FBX8).

Conclusions. The present study demonstrated that GSTP1 could enhance the expression of STAT3 to promote the proliferation, invasion and metastasis of CRC cells, which provides a potential therapeutic target for the clinical treatment of CRC.

Key words: metastasis, proliferation, colorectal cancer, glutathione S-transferase P1, signal transducer and activator of transcription 3 


\section{Background}

Following lung and breast cancer, colorectal cancer (CRC) is the $3^{\text {rd }}$ most common cancer worldwide. ${ }^{1}$ In many cases, primary CRC can be treated with surgery, chemotherapy and radiotherapy. However, it is limited in the restriction of the development of metastatic disease. ${ }^{2}$ Therefore, there is an urgent need to further clarify the molecular mechanism of CRC tumorigenesis and pathogenesis.

Glutathione S-transferases (GSTs) (the Enzyme Commission (EC) number: EC 2.5.1.18) are phase II metabolic enzymes, ${ }^{3}$ which play essential roles in xenobiotic biotransformation, ${ }^{4}$ drug metabolism, ${ }^{5}$ protection against oxidative stress, and regulating cell proliferation and signaling pathways. ${ }^{6,7}$ As an isoform of GST, glutathione S-transferase P1 (GSTP1) is a significant regulator of cell signaling in response to stress, hypoxia, growth factors, and other stimuli. ${ }^{8}$ The GSTP1 is overexpressed in various human cancers, including gastric cancer, pancreatic cancer and bladder cancer.,10 The GSTP1 is also involved in the proliferation and invasion of tumor cells; the overexpression of GSTP1 promotes the proliferation and inhibits the apoptosis in head and neck squamous cell carcinoma (HNSCC), ${ }^{11}$ whereas it inhibits the proliferation of T24 cells in bladder cancer. ${ }^{12}$ A recent study suggested that GSTP1 might be applied as an essential biomarker of liquid biopsy. ${ }^{13}$

The signal transducer and activator of transcription $(S T A T)$ family proteins are phosphorylated via Janus kinases $(J A K S)$ in response to the binding of growth factors or cytokines to their corresponding receptors. ${ }^{14-16}$ These factors are known to stimulate the activation of intracellular STAT proteins, which are phosphorylated and dimerized, and subsequently translocated to the nucleus for the transactivation of several genes involved in numerous cellular processes. ${ }^{17}$ The persistent activation of STAT3 has been observed in multiple human malignancies, including various CRC stages. ${ }^{18-20}$ Furthermore, a high expression of STAT3 alters the cell cycle ${ }^{21,22}$ and inhibits the apoptosis by upregulating anti-apoptotic signaling ${ }^{23,24}$ in inflammation-associated CRC and other human cancers. ${ }^{25}$ Besides, GSTP1 negatively regulates STAT3 activation in epidermal growth factor (EGF) signaling, and is also a regulator of the cell cycle via EGF signaling in human hepatocellular carcinoma (HCC). ${ }^{8}$ However, the regulatory mechanisms between GSTP1 and STAT3 in the progression of CRC remain unknown.

Our previous studies identified that the loss of F-box only protein $8(F B X 8)$ was associated with patients' poor survival in $\mathrm{HCC}$, gastric cancer and CRC. ${ }^{26-28}$ The FBX8 is a metastatic suppressor downstream of miR-223 and targets the degradation of mammalian target of rapamycin (mTOR) in CRC. ${ }^{27}$ We also found that $F B X 8$ inhibited the proliferation, invasion and metastasis of CRC by promoting the degradation of GSTP1. Simultaneously, we confirmed that GSTP1 could be used as an effective marker to predict the prognosis of CRC. ${ }^{29}$

\section{Objectives}

Our present study revealed that the overexpression of GSTP1 could promote the proliferation, invasion and metastasis of CRC cells by upregulating STAT3, and that $F B X 8$ could regulate this function.

\section{Materials and methods}

\section{Samples collection and ethical approval}

A total of 20 paraffin-embedded CRC primary tumor samples were obtained from the Department of Pathology of Nanfang Hospital, Southern Medical University, Guangzhou, China. Each sample was diagnosed with primary CRC: tubular adenocarcinoma (in 14 samples); papillary adenocarcinoma (1); mucinous adenocarcinoma (3); and signet-ring cell carcinoma (2). Fresh CRC tissues were collected immediately after resection from 8 patients who underwent CRC resection without prior radiotherapy and chemotherapy at the Department of General Surgery of Nanfang Hospital in 2019. All aspects of the study complied with the principles of the Declaration of Helsinki and the study was approved by the Nanfang Hospital Research Ethics Committee. No informed consent was required, as the data were analyzed anonymously. Paraffin-embedded mice CRC tumor samples used in the study were prepared in our previous experiments. ${ }^{29}$ The research related to the use of animals complied with all relevant national regulations and institutional policies for the care and use of laboratory animals.

\section{Immunohistochemistry}

The CRC human and animal tissue samples, which were formalin-fixed and paraffin-embedded, were dewaxed and rehydrated, and $3 \%$ hydrogen peroxide $\left(\mathrm{H}_{2} \mathrm{O}_{2}\right.$; ZSGB-Bio, Beijing, China) was used to eliminate endogenous peroxidase. The antigens on the tissue slides were retrieved with $0.01 \mathrm{M}$ sodium citrate buffer ( $\mathrm{pH}$ 6.0) by microwave oven boiling for $5 \mathrm{~min}$. After blocking the nonspecific antigens of the tissues with $5 \%$ goat serum (ZSGB-Bio) for $1 \mathrm{~h}$ at room temperature, the anti-GSTP1 (1:200; Abcam, Cambridge, UK) or anti-STAT3 (1:100; Abcam) antibody was diluted in an appropriate proportion and incubated overnight at $4^{\circ} \mathrm{C}$. The tissue slides were incubated with the horseradish egg protein rabbit secondary antibody (ZSGB-Bio) or the murine secondary antibody (ZSGBBio) for $90 \mathrm{~min}$ at room temperature. Streptavidin was labeled with appropriate horseradish peroxidase (HRP; ZSGB-Bio), and incubated for $30 \mathrm{~min}$ at room temperature. The 3,3'-diaminobenzidine (DAB; ZSGB-Bio) color developer was needed for microscopic observations.

Staining was scored in a double-blind manner by 2 researchers with a score of 0 (representative negative), 1 (weak), 
2 (medium), and 3 (strong). Depending on the percentage of the stained area relative to the total cancerous tissue area or blood vessel area, the staining range was divided as follows: 0 points $(0 \%)$; 1 point $(1-25 \%) ; 2$ points $(26-50 \%) ; 3$ points (51-75\%); and 4 points (76-100\%). The sum of the dyeing strength and range was taken as the final dyeing value $(0-7)$ : $(-)$ total score $<3$ points; $(+)$ total score 3 points; $(++)$ total score 4 points; and $(+++)$ total score $\geq 5$ points, in which $(-)$ or $(+)$ form the low-expression groups, whereas $(++)$ and $(+++)$ are the high-expression groups.

\section{Co-immunoprecipitation}

In brief, the extracts of SW620 cells were blocked with immunoglobulin G (IgG) or protein A/G agarose (BioWorld, Visalia, USA) for $2 \mathrm{~h}$ at $4^{\circ} \mathrm{C}$ to remove nonspecific protein binding, and then incubated with anti-FBX8 (Abcam, Cambridge, UK) or anti-GSTP1 overnight at $4^{\circ} \mathrm{C}$. Protein A/G Agarose was separated by centrifugation $(2500 \mathrm{rpm})$ at $4{ }^{\circ} \mathrm{C}$. After blocking with $5 \%$ skim milk for $1 \mathrm{~h}$ at room temperature, polyvinylidene difluoride (PVDF) membranes (Roche Applied Science, Penzberg, Germany) were incubated with anti-GSTP1 (1:200) and anti-STAT3 (1:100) overnight at $4^{\circ} \mathrm{C}$. The protein bands were visualized by using an enhanced chemiluminescence (ECL) HRP kit (fluorescence detection (FD) bio-Femto (Yeasen, Shanghai, China).

\section{Immunofluorescence}

A total of $0.5 \times 10^{4}$ cells were seeded on confocal $\mathrm{NEST}^{\circledR}$ glass-bottom Petri dishes (NEST, Wuxi, China) for a 24-hour incubation. Then, the cells were fixed with $4 \%$ paraformaldehyde, permeabilized with $0.02 \%$ Triton$\mathrm{X}-100 / 1 \times$ phosphate-buffered saline $(\mathrm{PBS})$ and blocked in a blocking fluid $(1 \times \mathrm{PBS}+10 \%$ fetal bovine serum (FBS) and 1\% bovine serum albumin (BSA)) (Thermo Fisher Scientific, Waltham, USA). The Petri dishes were incubated with anti-GSTP1 (1:200) and anti-STAT3 (1:100) overnight at $4^{\circ} \mathrm{C}$. The secondary antibodies conjugated to Alexa Fluor ${ }^{\circledR} 488$ or 594 (Invitrogen, Waltham, USA) were added to the incubated cells for $2 \mathrm{~h}$ at room temperature. The 4',6-diamidino-2-phenylindole (DAPI) was used to stain the cell nucleus. Confocal images were taken with the use of an inverted fluorescence microscope (Olympus Corp., Tokyo, Japan) and outputted by means of the PV10-ASW viewer software v. 1.7 (Olympus Corp.).

\section{Glutathione S-transferase pull-down assay}

We used GST-mediated pull-down assays (Thermo Fisher Scientific, Rockford, USA) to detect the interaction between the truncated GSTP1 and STAT3 in HCT116 and SW620 cell lines. Firstly, recombinant GST-STAT3CCD (coiled-coil domain) (218-400), GST-STAT3-DBD (DNA-binding domain) (401-564), GST-STAT3-linker
(565-663), and GST-STAT3-SH2 (Src homology 2 domain) (664-768) proteins were expressed and purified. Secondly, the purified GST-STAT3-CCD (218-400), GSTSTAT3-DBD (401-564), GST-STAT3-linker (565-663), and GST-STAT3-SH2 (664-768) fragments were bound to glutathione resin as a GST-fusion protein and incubated with anti-GSTP1 for $2 \mathrm{~h}$ at $4^{\circ} \mathrm{C}$. Thirdly, the complex was washed with the assay buffer and eluted with $5 \mathrm{mM}$ reduced glutathione, and then the bound protein complexes were disrupted. Finally, the proteins were separated by means of sodium dodecyl sulfate-polyacrylamide gel electrophoresis (SDS-PAGE) and detected with a western blotting assay.

\section{Plasmid and siRNA transfection}

The cells were transfected with plasmids or siRNA for STAT3 or GSTP1, respectively, with Lipofectamine ${ }^{\circledR} 3000$, as per the manufacturer's instructions (Thermo Fisher Scientific, Waltham, USA).

\section{Cell proliferation assay (CCK-8)}

The medium in a 96-well plate was replaced with $100 \mu \mathrm{L}$ of the complete medium and Cell Counting Kit-8 (CCK-8) reagent mixture (9:1), and the plate was incubated in $5 \%$ $\mathrm{CO}_{2}$ for $2-4 \mathrm{~h}$ at $37^{\circ} \mathrm{C}$. The optical density (OD) value at $450 \mathrm{~nm}$ for each well was detected with an enzyme labeling instrument (BioTek, Winooski, USA), and the OD value was adjusted to zero based on the blank control group. The cell proliferation curve was plotted with the mean value of 5 multiple holes in each group.

\section{Cell invasion assays in vitro}

Cells invasion assays in vitro were performed to assess the cell invasion ability. In brief, $200 \mu \mathrm{L}$ of a serum-free medium containing $5 \times 10^{4}$ cells was added to the upper chamber with the Corning ${ }^{\circledR}$ BioCoat ${ }^{\mathrm{TM}}$ Matrigel (Corning Life Sciences, New York, USA) and allowed to invade toward the lower chamber with $10 \%$ FBS. After incubation for an appropriate period of time at $37^{\circ} \mathrm{C}$, the cells were fixed with methanol for $30 \mathrm{~min}$ and stained with crystal violet solution for $20 \mathrm{~min}$. The migrated cells were photographed and counted in 6 random regions with an inverted microscope (Olympus Corp.).

\section{Western blotting}

During immunoblotting, the proteins were extracted with a lysis buffer, and then quantified by means of a bicinchoninic acid (BCA) protein assay kit (Beyotime, Shanghai, China). Equivalent amounts of cell lysates were separated using SDS-PAGE and transferred to PVDF membranes. The membranes were immunoblotted overnight at $4^{\circ} \mathrm{C}$ with anti- $\beta$-actin antibodies (1:1000; Proteintech, Chicago, 
USA), GSTP1 (1:200; Abcam, London, England), STAT3 (1:200; Abcam), GST (1:1000; Abcam), followed by appropriate second antibodies. The bands were visualized using the Pierce ${ }^{\mathrm{TM}}$ ECL western blotting substrate (Thermo Fisher Scientific). The immunoblotting image density was determined with gel densitometry (Bio-Rad Laboratories, Hercules, USA).

\section{Quantitative reverse transcription polymerase chain reaction (qRT-PCR) analysis}

The RNA of the cultured cells and the CRC tissues were extracted using the TRIzol ${ }^{\circledR}$ reagent (Invitrogen). The RNA expression level of GSTP1 was detected with the use of ABI PRISM 7500 Fast Real-Time PCR System (Thermo Fisher Scientific). The relative $\mathrm{mRNA}$ levels were calculated using the comparative $\mathrm{Ct}$ method $(\Delta \Delta \mathrm{Ct})$. The primer sequences for qRT-PCR were as follows:

- GSTP1: Fwd: CGG GGT ACC ATG CCG CCC TAC ACC GT; Rev: CCG CTC GAG TCA CTG TTT CCC GTT GCC ATT;

- STAT3: Fwd: CAG CAG CTT GAC ACA CGG TA; Rev: AAA CAC CAA AGT GGC ATG TGA.

\section{Cell culture and treatment}

All cell lines were obtained from American Type Culture Collection (ATCC; Manassas, USA). The human CRC cell lines (SW480, SW620, HCT116, and LOVO) were cultured in the Roswell Park Memorial Institute medium (RPMI) 1640 (high glucose) (Gibco Laboratories, Gaithersburg, USA) supplemented with 10\% FBS (Gibco Laboratories). The medium was supplemented with $100 \mu \mathrm{g} / \mathrm{mL}$ streptomycin and $100 \mathrm{U} / \mathrm{mL}$ penicillin $\left(\mathrm{Gibco}^{\mathrm{TM}}\right.$, Thermo Fisher Scientific, Waltham, USA), and the abovementioned cells were incubated at $37^{\circ} \mathrm{C}$ in a humidified chamber containing $5 \% \mathrm{CO}_{2}$. The stable knocking down GSTP1 cancer cells (SW620/shGSTP1 and HCT116/shGSTP1 cell lines) were from the previous study.

\section{Statistical analysis}

All statistical analyses were performed by means of GraphPad Prism v. 6.02 (GraphPad Software, San Diego, USA). The results are presented as mean (M) and standard deviation (SD) or median (Me) and 95\% confidence interval (95\% CI). Pearson's correlation coefficient was used to analyze the correlation between GSTP1 and STAT3. The oneway analysis of variance (ANOVA) was used to compare differences in the invasion ability of the 3 groups in invasion assays, and the two-way ANOVA to analyze differences in the proliferation ability of CRC cells at different time points between different groups. Before ANOVA, Levene's test for the equality of variances was used. A two-tailed $\mathrm{p}<0.05$ was considered statistically significant in all tests.

\section{Results}

The expression levels of GSTP1 and STAT3 in human CRC tissues are positively correlated

The STAT3, widely recognized as a cancer gene, is typically associated with a poor prognosis for various human malignancies, as it promotes cancer progression or metastasis. ${ }^{30-33}$ The direct interaction between GSTP1 and STAT3 can promote HCC progression, ${ }^{8}$ and our previous study found that GSTP1 could be ubiquitinated by $F B X 8$, thus inhibiting its function in promoting CRC proliferation, invasion and metastasis. ${ }^{29}$ Therefore, immunohistochemistry (IHC) was used to detect the expression of GSTP1 and STAT3 in 20 human CRC tissue samples. The results demonstrated that the expression levels of GSTP1 and STAT3 in human CRC tissues were positively correlated (Fig. 1A). Western blotting and qRT-PCR were used to detect the expression levels of GSTP1 and STAT3 in 8 paired fresh CRC tissue samples (Fig. 1B,C), and the results of these 2 experiments showed that the expression patterns of GSTP1 and STAT3 were highly correlated. After statistical analysis, it turned out that the expression of GSTP1 was positively correlated with the expression of STAT3 in the paired fresh CRC tissue samples, as shown in Fig. 1D (Pearson's correlation analysis, two-tailed test, $\mathrm{r}^{2}=0.8781, \mathrm{p}=0.0006$ ).

\section{The overexpression of GSTP1 promotes the proliferation, invasion and metastasis of CRC cells depending on STAT3}

The expression of GSTP1 was positively correlated with the expression of STAT3. Taking into account previous studies, it was predicted by authors that GSTP1 might play a role in CRC progression by regulating STAT3. Therefore, the SW620 and HCT116 cell lines which stably knockdown GSTP1 were constructed (Fig. 2A). Next, STAT3 was overexpressed in the above 2 cell lines; the results showed that the overexpression of STAT3 was successful (Fig. 2A). Therefore, the SW620/shGSTP1/STAT3 and HCT116/shGSTP1/STAT3 cell lines were used to perform relevant recovery experiments. The results showed that the overexpression of STAT3 could significantly promote the invasion (Fig. 2B) and proliferation (Fig. 2C) of cells in the GSTP1-knockdown group in vitro. Besides, the expression levels of STAT3 and GSTP1 were detected in subcutaneous tumors (Fig. 3A), in situ implants (Fig. 3B) and liver metastases (Fig. 3C) of CRC in the nude mouse tissues obtained from a previous study. The results demonstrated that in these 3 tumor tissues, the expression of STAT3 was significantly upregulated in the GSTP1 overexpressed group, which indicated that the expression levels of GSTP1 and STAT3 in the mouse tissue samples were consistent. 
A
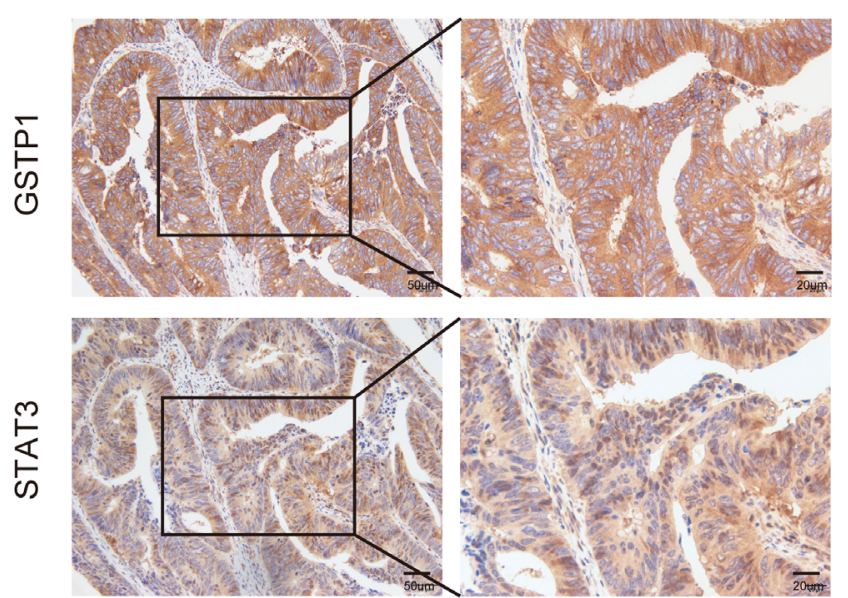

low expression
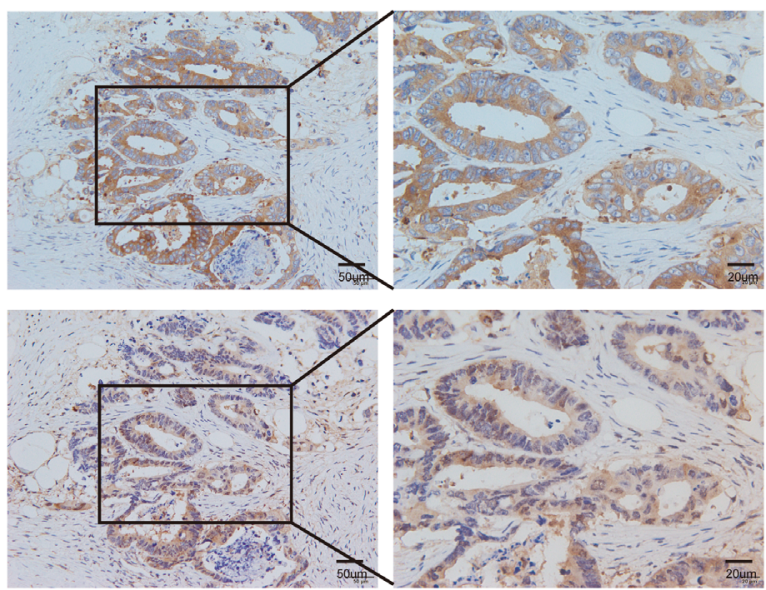

B

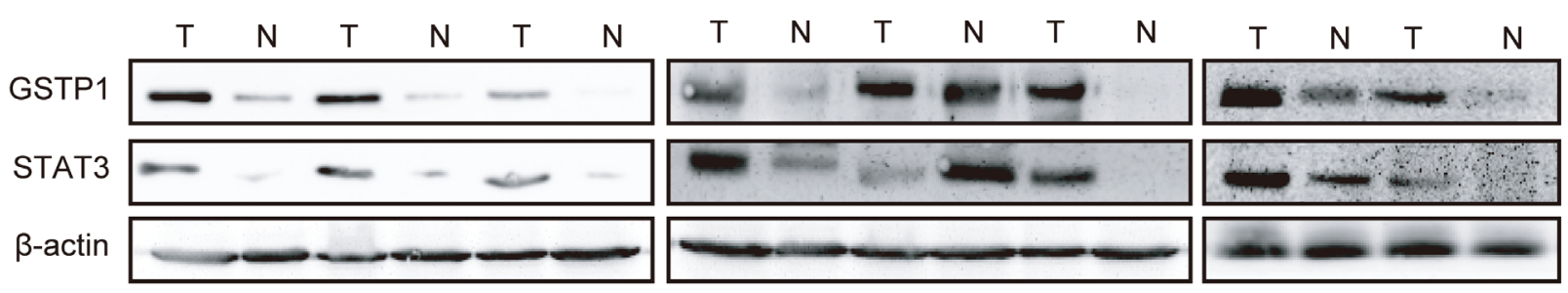

C

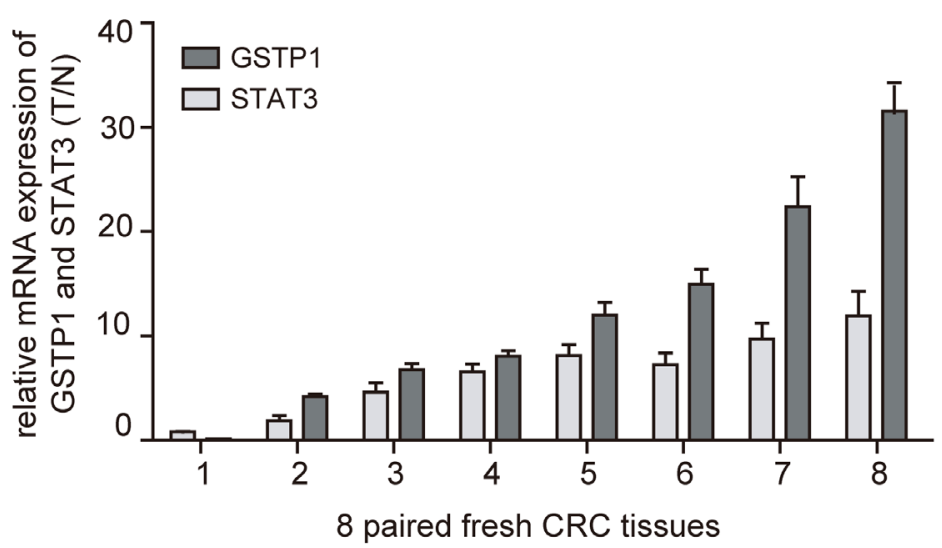

D

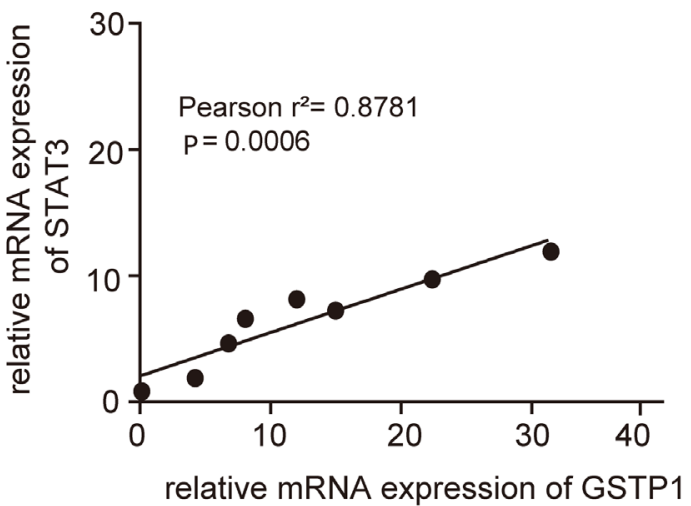

Fig. 1. Expression levels of glutathione S-transferase P1 (GSTP1) and signal transducer and activator of transcription 3 (STAT3) in human colorectal cancer (CRC) tissues are positively correlated

A. The expression levels of GSTP1 and STAT3 in CRC tissues were detected by means of immunohistochemistry (IHC). Scale bars represent 50 um (left) and 20 m (right); B. The western blot analysis was performed to detect the expression levels of GSTP1 and STAT3 in 8 paired fresh tissue samples of human CRC; $C$. The relative mRNA expression levels of GSTP1 and STAT3 in 8 paired fresh tissue samples of human CRC were detected by means of the quantitative reverse transcription polymerase chain reaction ( $\mathrm{QRT}$-PCR) analysis. All samples were tested in triplicate. Bars represent mean \pm standard deviation (M $\pm \mathrm{SD}$ ); D. The analysis of correlation between GSTP1 and STAT3 was performed using Pearson's correlation coefficient (two-tailed test).

\section{GSTP1 and STAT3 can directly bind to and regulate each other}

It was further hypothesized that GSTP1 could interact with STAT3 in CRC cells. Therefore, the co-immunoprecipitation (co-IP) and immunofluorescence (IF) analyses were performed to identify the interaction between the 2 proteins. As expected, the existence of GSTP1 in the immunoprecipitates obtained with an antibody against STAT3 was successfully detected (Fig. 4A). The IF results demonstrated that
GSTP1 and STAT3 exhibited co-localization in the cytoplasm of the SW620 cells (Fig. 4B). The present study cloned 4 truncated constructs of STAT3: CCD (218-400); DBD (401-564); linker (565-663); and SH2 (664-768) (Fig. 4C), and then identified an interaction between the CCD domain of STAT3 and GSTP1 by means of a GST pull-down assay (Fig. 4C). It turned out that the CCD domain of STAT3 was essential for the interaction with GSTP1.

Thus, it was examined whether GSTP1 could activate the STAT3 signaling pathway in CRC cells, and then it was 
A
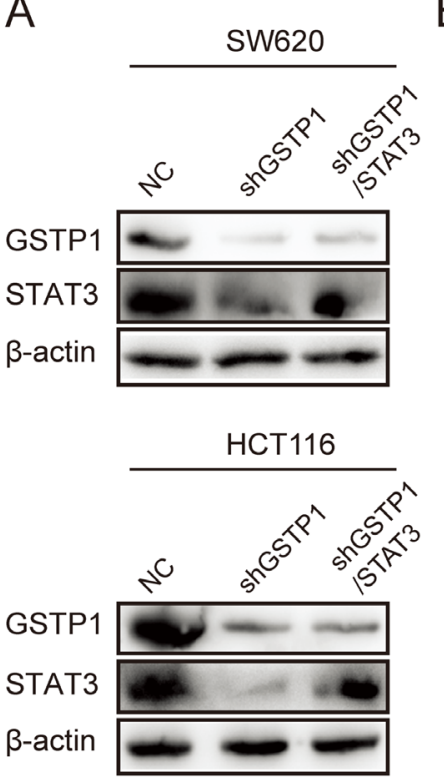

B
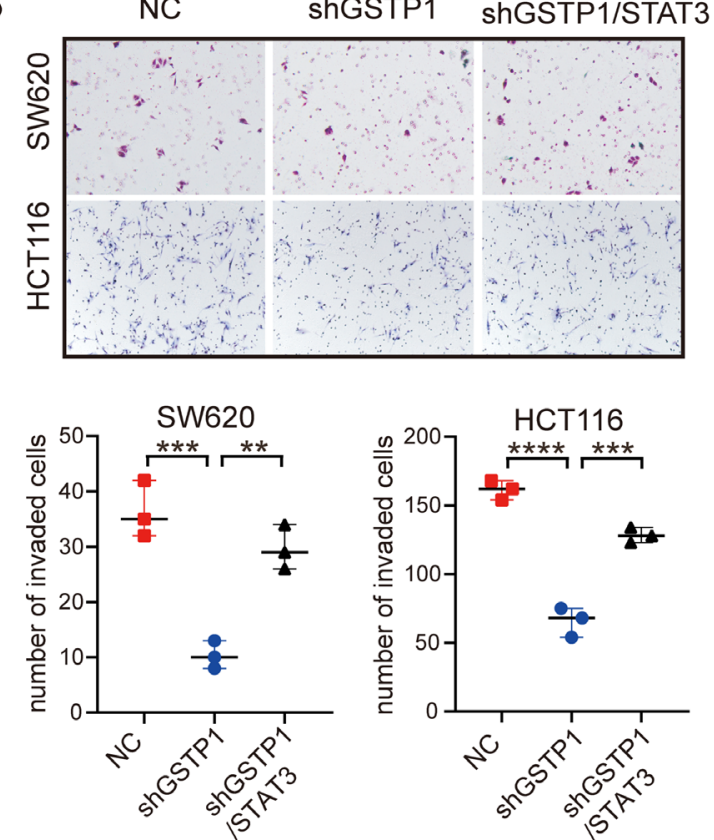

C
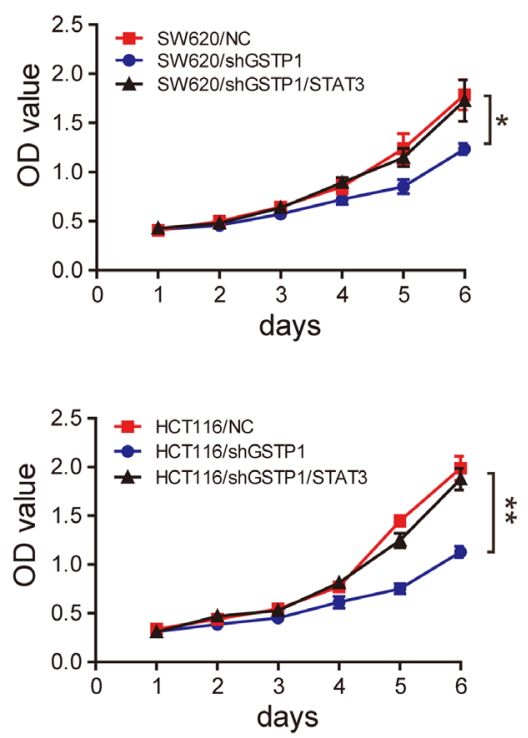

Fig. 2. Overexpression of signal transducer and activator of transcription 3 (STAT3) can reverse the inhibition of proliferation and invasion induced by downregulating glutathione S-transferase P1 (GSTP1)

A. The western blot analysis was performed to detect the effects of the overexpression of STAT3 on SW620/shGSTP1 and HCT116/shGSTP1 cells; B. The effect of the overexpression of STAT3 on SW620/shGSTP1 and HCT116/shGSTP1 cell invasion was assessed with an invasion assay. Scale bars represent $50 \mathrm{\mu m}$. All samples were tested in triplicate. The statistical analysis was performed with the one-way analysis of variance (ANOVA). Point charts represent median (Me) and 95\% confidence interval $(95 \% \mathrm{Cl}) ;{ }^{* *} \mathrm{p}<0.005 ;{ }^{* * *} \mathrm{p}<0.0005 ;{ }^{* * * *} \mathrm{p}<0.0001$; C. The effect of the overexpression of STAT3 on SW620/shGSTP1 and HCT116/shGSTP1 cell proliferation was detected with a cell counting-8 (CCK-8) assay. All samples were tested in triplicate. The statistical analysis was performed with the two-way ANOVA. Bars represent mean \pm standard deviation (SD); OD - optical density; NC - negative control; ${ }^{*} \mathrm{p}<0.05 ;{ }^{* *} \mathrm{p}<0.005$.

identified that the exogenous expression of GSTP1 further increased the protein expression of phosphorylated (p)STAT3, STAT3 and downstream STAT3 targets (cyclin D1 and CDC25A) in the SW480 cells (Fig. 4D). However, there was no appreciable effect on the upstream components of the STAT3 signaling pathway, such as JAK2 and p-JAK2 (Fig. 4D). By contrast, the depletion of GSTP1 in the SW620 cells decreased the levels of p-STAT3, STAT3, cyclin D1, and CDC25A (Fig. 4D). Notably, this regulation was not one-way; the ectopic expression of STAT3 could also upregulate the protein level of GSTP1 and induce higher levels of downstream STAT3 targets (cyclin D1 and CDC25A). Simultaneously, silencing STAT3 could decrease the expression levels of GSTP1, cyclin D1 and CDC25A, but not the expression of p-JAK2 (Fig. 4E). Also, when AG490 $(100 \mu \mathrm{M})$ was used to block the JAK2-STAT3 pathway, a significant decrease was observed in the expression of JAK2, p-JAK2, STAT3, p-STAT3, and GSTP1 24 h after treating the LoVo cells (Fig. 4F).

\section{The interaction between GSTP1 and STAT3 is regulated by FBX8}

As a downstream target of $F B X 8, G S T P 1$ can interact with STAT3. Therefore, it was predicted that $F B X 8$ could regulate the interaction between GSTP1 and STAT3. The coIP analysis demonstrated that there was an interaction between GSTP1 and STAT3 in the SW620 cell line, with a high endogenous expression of $F B X 8$ (Fig. 5A). However, after adding AG490, a specific inhibitor of JAK2, the signal transduction of the JAK2 pathway was inhibited, resulting in a significant decrease in the expression of STAT3 in the SW620 cell line. In comparison with the group without AG490, the STAT3 that GSTP1 could bind to was also significantly reduced (Fig. 5B). Simultaneously, in the SW480 cell line with the overexpression of $F B X 8$, the expression of STAT3 detected with a co-IP assay was significantly reduced as compared to the control group (Fig. 5C). These results indicated that FBX8 was a suppressive factor for the combination of GSTP1 and STAT3.

Figure 6 is the schematic diagram of the role of GSTP1, STAT3 and FBX8 in CRC.

\section{Discussion}

Previously, we identified GSTP1 as a downstream target of $F B X 8$ using the co-IP and mass spectrometry analyses, and confirmed that GSTP1 could promote the proliferation, invasion and metastasis of CRC. ${ }^{29}$ Besides, it has been found that GSTP1 could regulate STAT3 to affect the development of HCC. ${ }^{8}$ Therefore, we hypothesized that GSTP1 might be involved in the progression of CRC by regulating STAT3. 
A

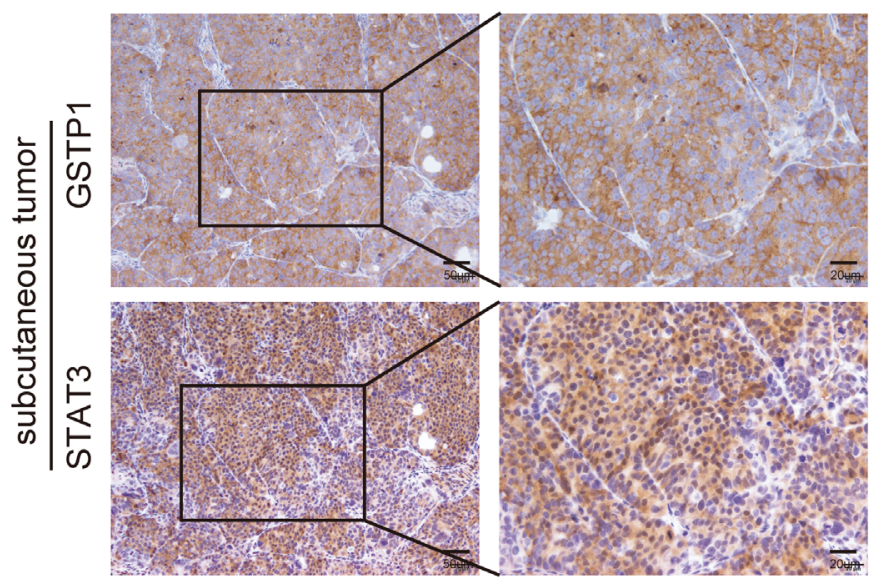

B

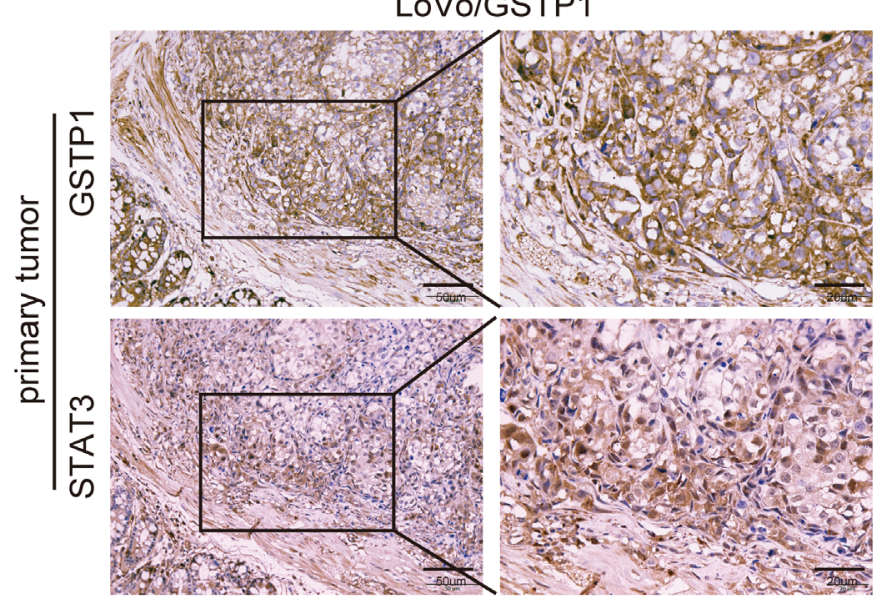

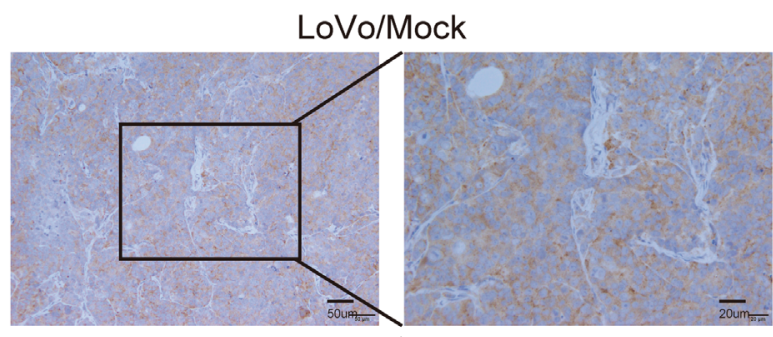

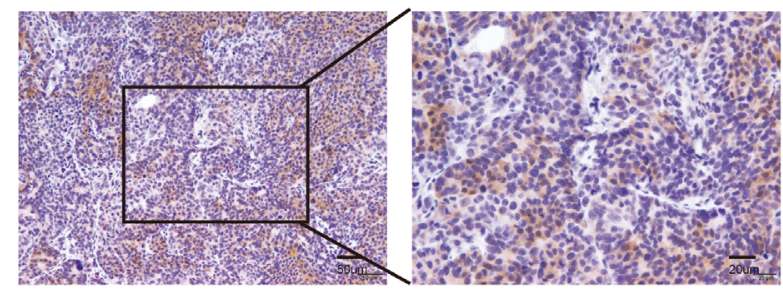

LoVo/Mock
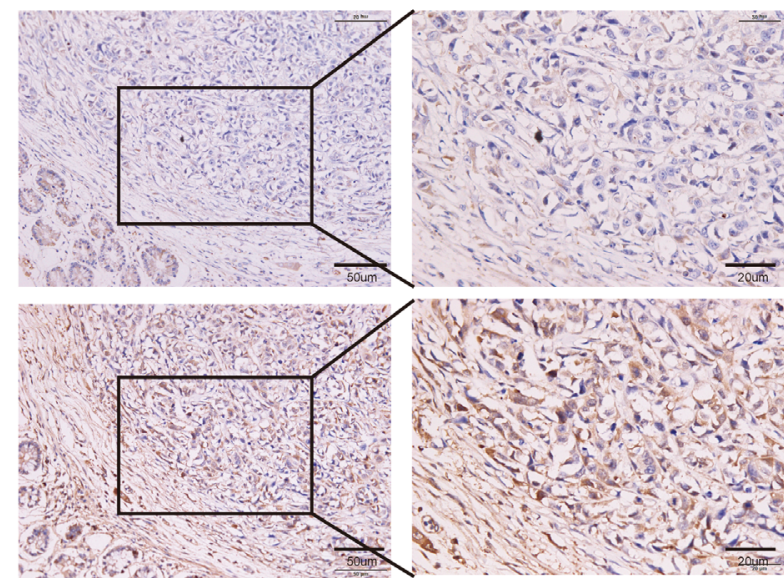

LoVo/Mock
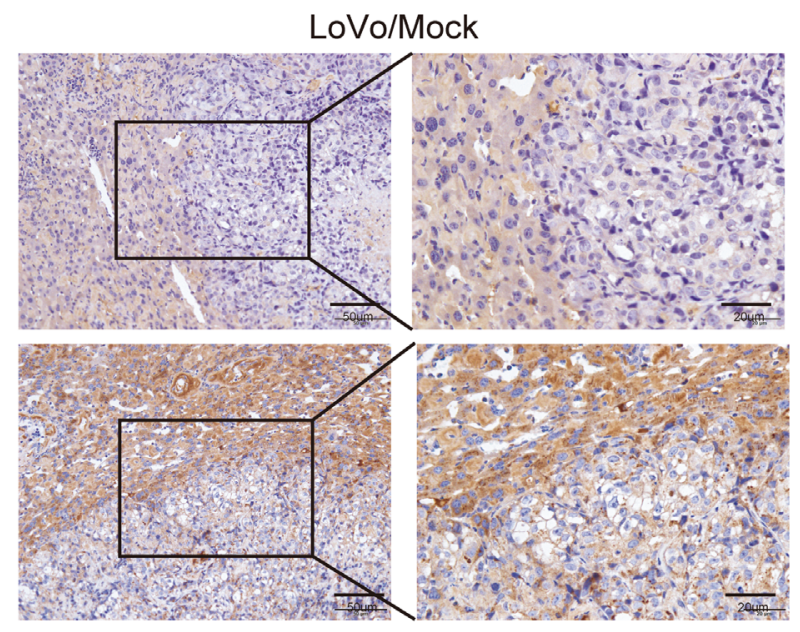

C

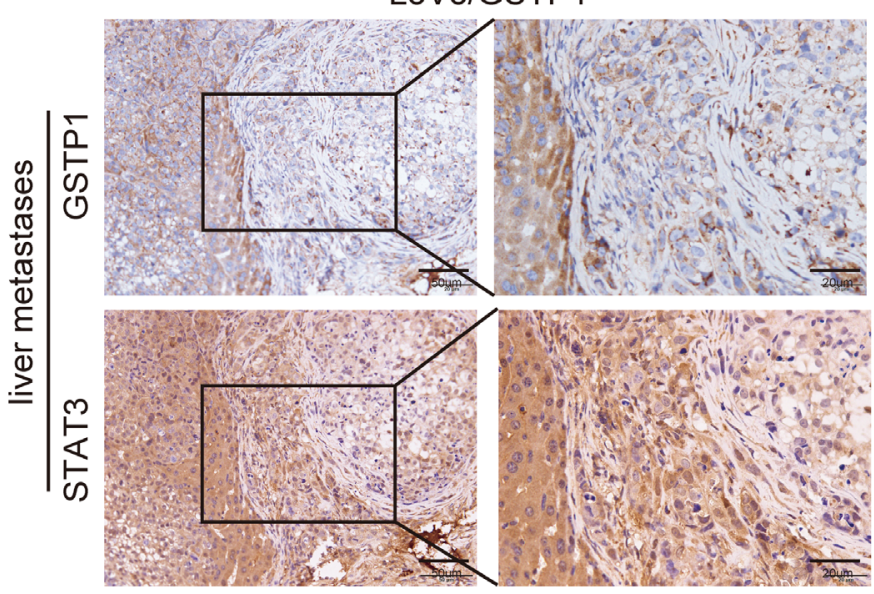

Fig. 3. Overexpression of glutathione S-transferase P1 (GSTP1) can upregulate the expression of signal transducer and activator of transcription 3 (STAT3) in vivo

A. The expression of GSTP1 and STAT3 in subcutaneous tumors in nude mice was detected by means of immunohistochemistry (IHC). Scale bars represent $50 \mu \mathrm{m}$ (left) and $20 \mu \mathrm{m}$ (right); B. The expression of GSTP1 and STAT3 in the orthotopic implantation of colorectal cancer (CRC) in nude mice was detected by means of IHC. Scale bars represent $50 \mu \mathrm{m}$ (left) and $20 \mu \mathrm{m}$ (right); C. The expression of GSTP1 and STAT3 in liver metastases of CRC in nude mice was detected by means of IHC. Scale bars represent $50 \mu \mathrm{m}$ (left) and $20 \mu \mathrm{m}$ (right).

The present study detected GSTP1 and STAT3 in human CRC tissue samples and found that the expression of GSTP1 was positively correlated with the expression of STAT3. This result revealed that GSTP1 might be able to regulate the expression of STAT3, and thus plays a role in the progression of CRC. Recent evidence suggests that GSTP1 is involved in tumor cell proliferation and invasion; the overexpression of GSTP1 increases cell proliferation 
A

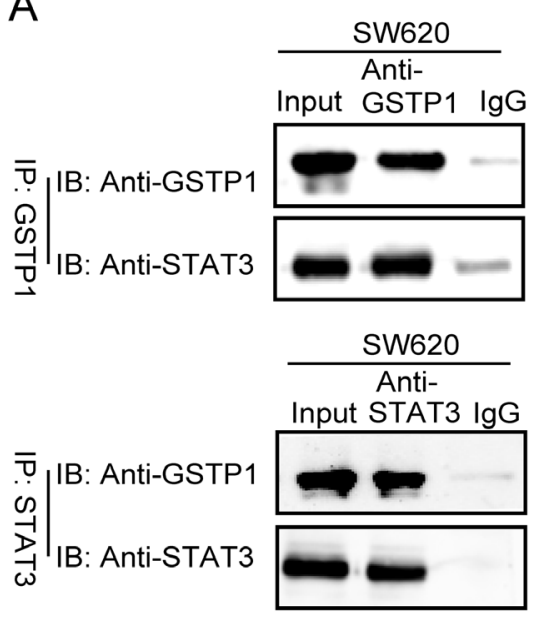

$\mathrm{D}$

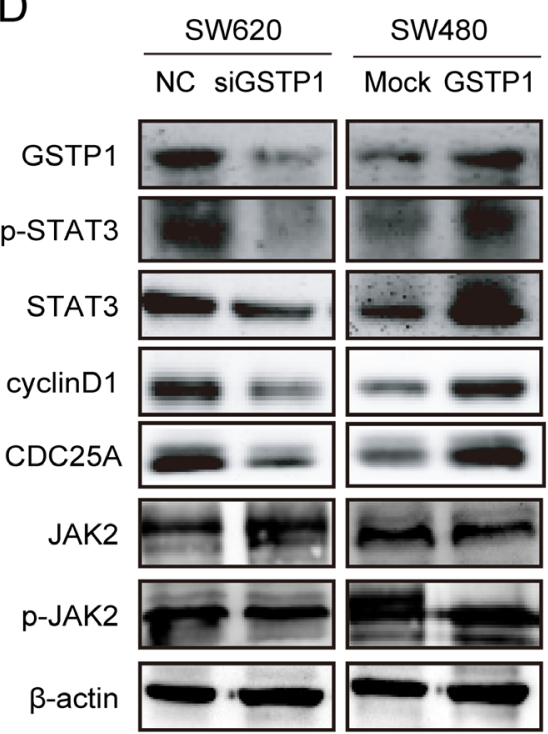

B

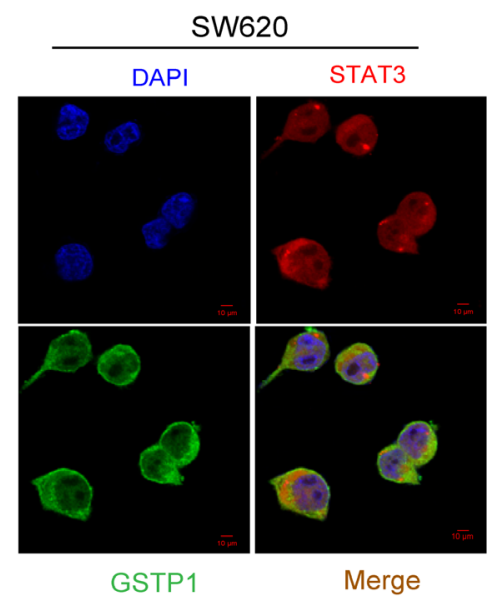

C

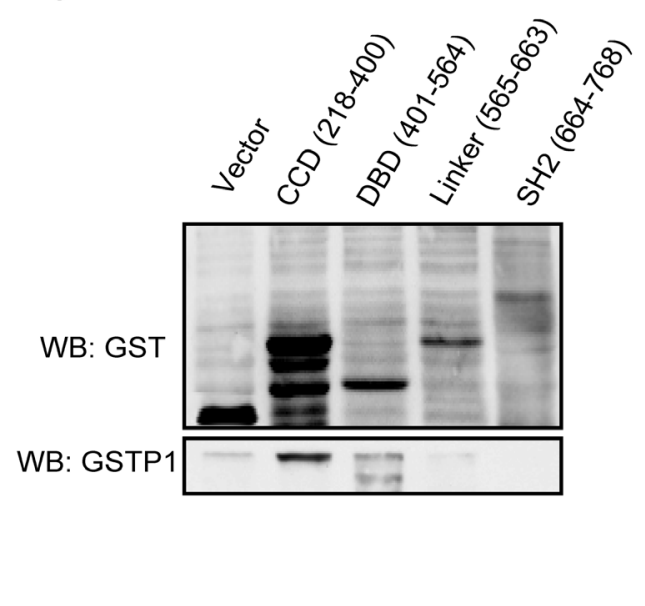

$\mathrm{F}$

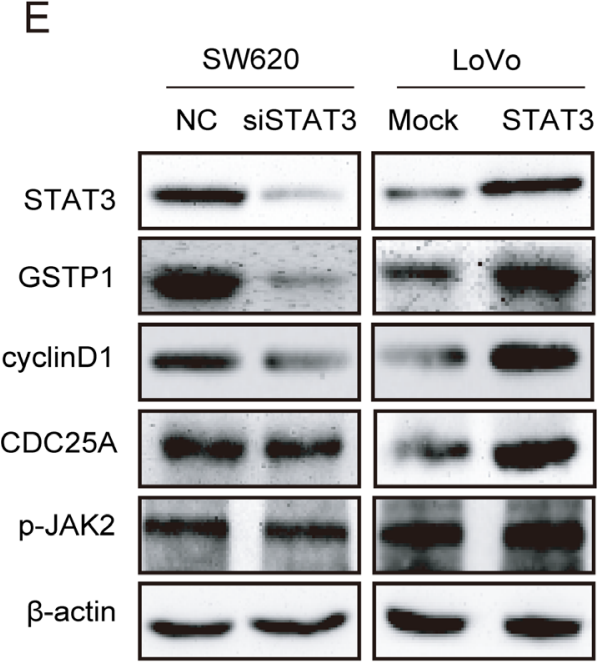

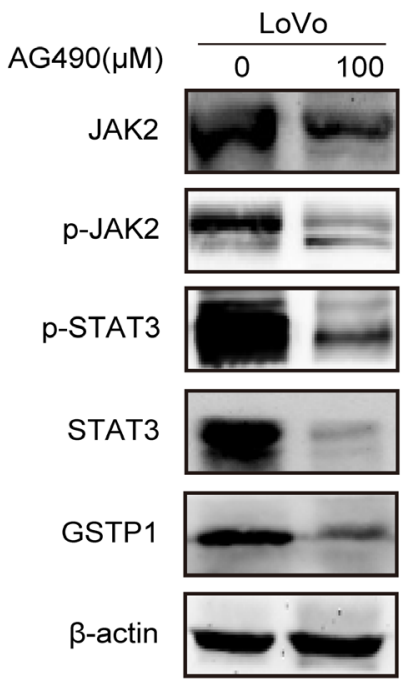

Fig. 4. Glutathione S-transferase P1 (GSTP1) and signal transducer and activator of transcription 3 (STAT3) can interact with each other

A. The interaction between GSTP1 and STAT3 was detected by means of a co-immunoprecipitation (co-IP) assay with the SW620 cell line; IB - immunoblotting; IgG - immunoglobulin G; B. The co-localization between GSTP1 and STAT3 was detected by means of an immunofluorescence (IF) assay with the SW620 cell line. Scale bars represent 10 um; DAPI - 4',6-diamidino-2-phenylindole; C. The direct interaction site between GSTP1 and STAT3 was detected by means of a GST pull-down assay; WB - western blotting; D. The western blot analysis was performed to detect the expression of Janus kinase (JAK)/STAT3 signaling pathway-related proteins in the SW620/siGSTP1 and SW480/GSTP1 cell lines; p-STAT3 - phosphorylated STAT3; p-JAK2 - phosphorylated JAK2; E. The western blot analysis was performed to detect the expression of JAK/STAT3 signaling pathway-related proteins in the SW620/siSTAT3 and LoVo/STAT3 cell lines; F. The western blot analysis was performed to detect the expression of GSTP1 and JAK/STAT3 signaling pathway-related proteins following the AG490 treatment of the LoVo cells.

in HNSCC. ${ }^{11}$ By comparison, GSTP1 can arrest tumor cells in the G0/G1 phase and upregulate the expression of p21 in bladder cancer. ${ }^{12}$ The present study investigated the effect of GSTP1 on the proliferation and invasion of CRC cells in vitro by recovery experiments. The results demonstrated that the overexpression of STAT3 could significantly promote the proliferation and invasion of CRC cells after GSTP1 downregulation. This indicated that GSTP1 enabled the proliferation and invasion of CRC cells depending on STAT3. Besides, the IHC results of subcutaneous tumors, in situ implanted tumors and liver metastases of CRC in mice also confirmed the conclusion mentioned above.

In terms of mechanism, this study demonstrated that
GSTP1 and STAT3 could form a complex, and GSTP1 upregulation leads to the activation of the STAT3 pathway. Meanwhile, STAT3 can positively regulate the protein expression of GSTP1. The STAT3 is phosphorylated via JAK, then dimerized and subsequently translocated to the nucleus for the transactivation of several genes involved in numerous cellular processes. ${ }^{14-17}$ Besides, the overexpression of STAT3 can affect the cell cycle ${ }^{21,22}$ or inhibit the apoptosis by enhancing anti-apoptotic signaling ${ }^{23,24}$ in CRC.

Therefore, identifying the association between GSTP1 and the STAT3 pathway is a meaningful way to illustrate the molecular mechanisms of GSTP1 in CRC. This study confirmed the interaction between GSTP1 and STAT3 and 
A

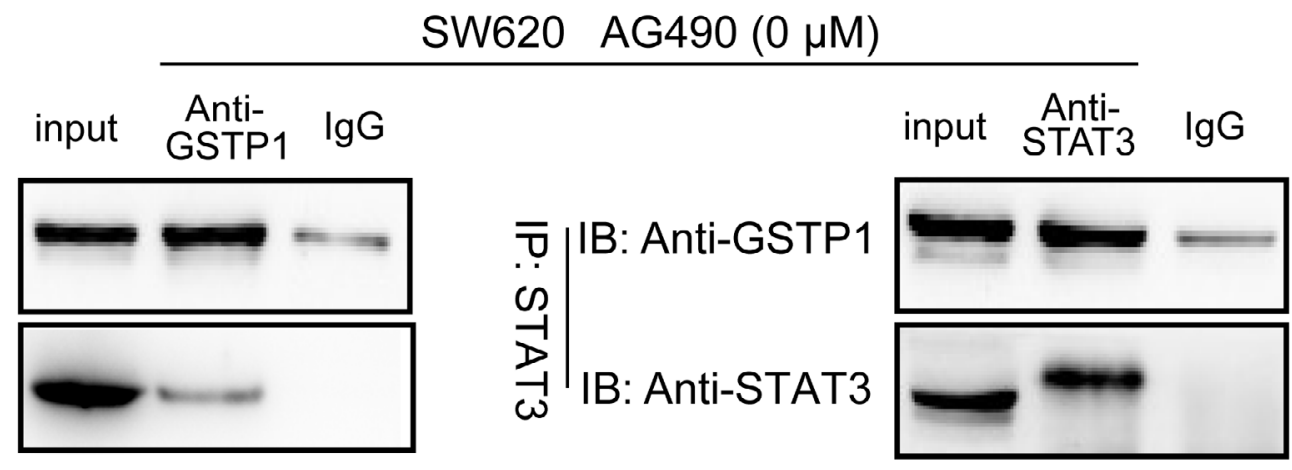

B

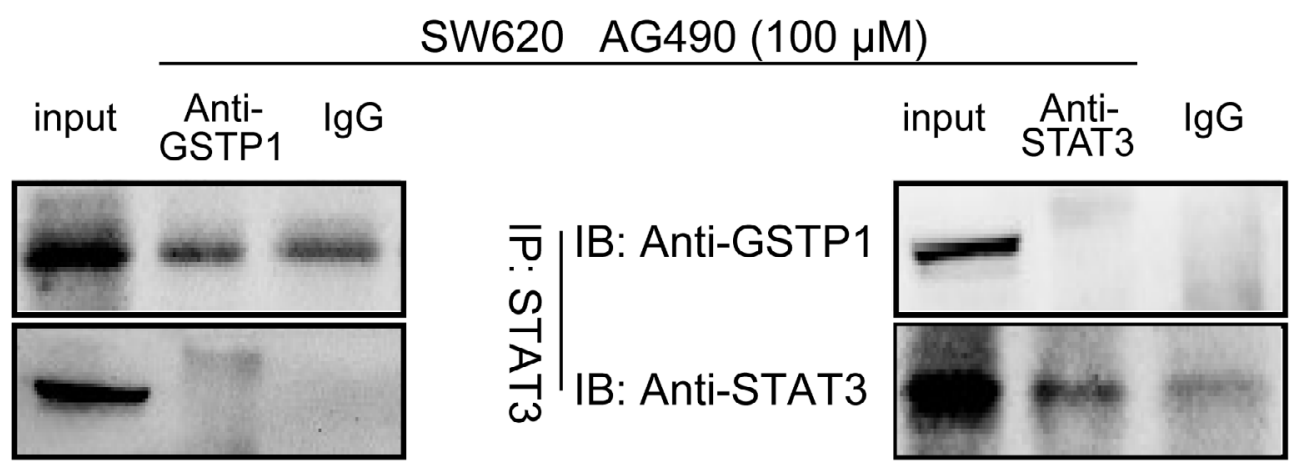

C
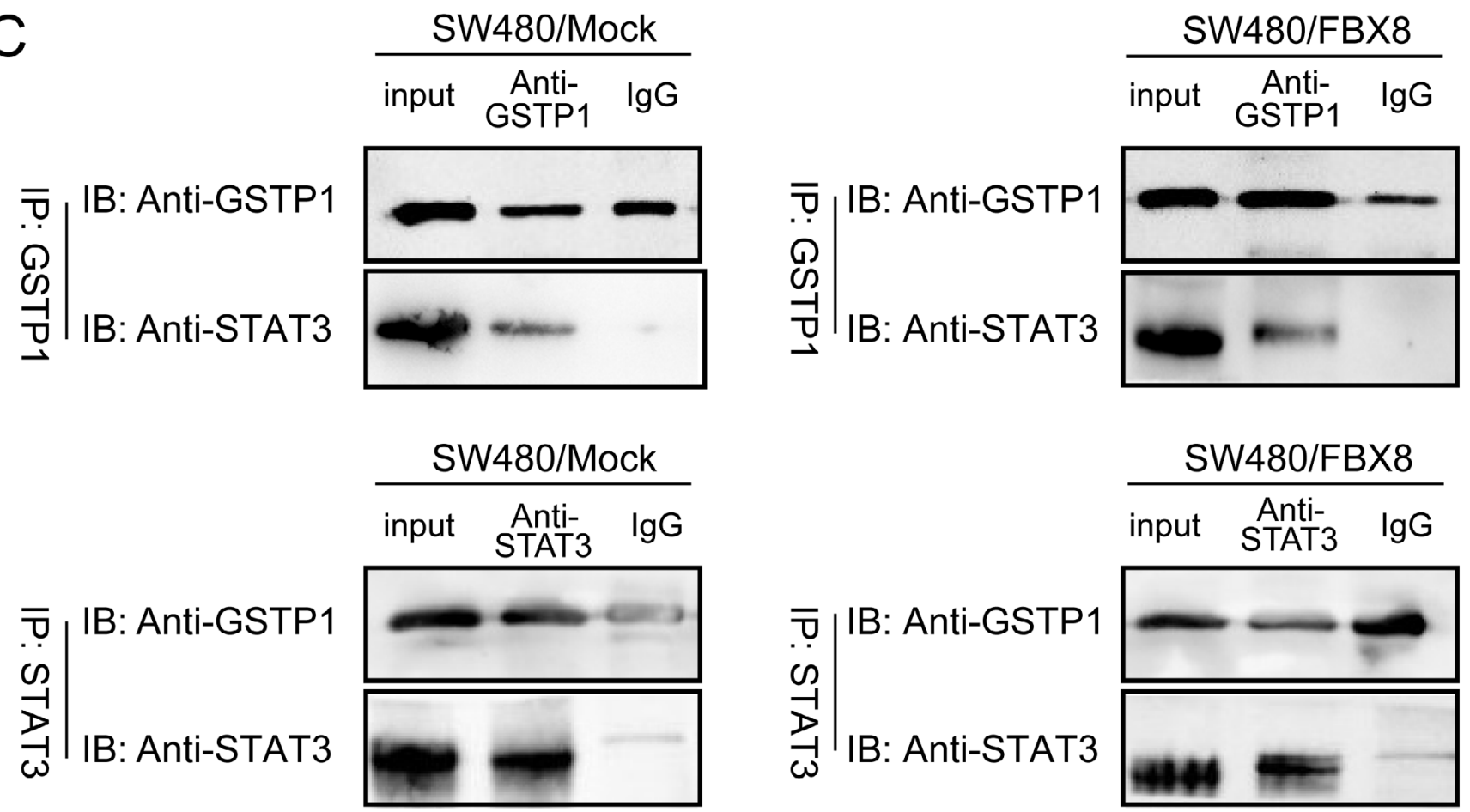

Fig. 5. Interaction between glutathione S-transferase P1 (GSTP1) and signal transducer and activator of transcription 3 (STAT3) is regulated by F-box only protein 8 (FBX8)

A. The interaction between GSTP1 and STAT3 was detected by means of a co-immunoprecipitation (co-IP) assay in the SW620 cell line; B. The interaction between GSTP1 and STAT3 was detected by means of a co-IP assay in the SW620 cell line after AG490 treatment; C. The interaction between GSTP1 and STAT3 was detected by means of a co-IP assay in the SW480/FBX8 cells.

proved that GSTP1 positively regulates the STAT3 signaling pathway, leading to changes in target genes, such as pSTAT3 and STAT3, cyclin D1, and CDC25A. The STAT3
siRNA significantly abolished increases in STAT3, cyclin D1 and CDC25A and decreased the protein expression of GSTP1, but there was no change in p-JAK2. Exogenous 


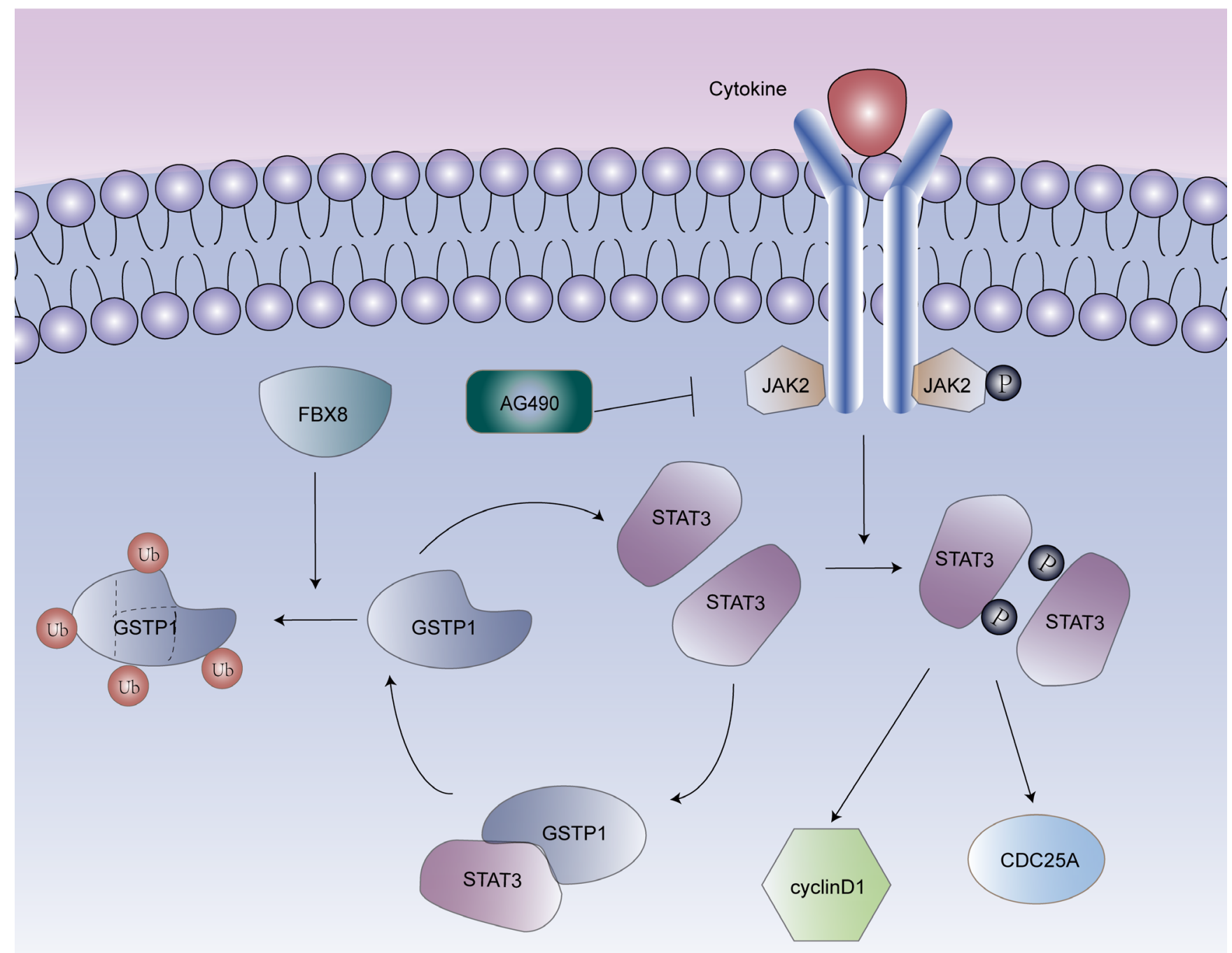

Fig. 6. Schematic diagram of the role of glutathione S-transferase P1 (GSTP1) and signal transducer and activator of transcription 3 (STAT3) in colorectal cancer (CRC)

STAT3 exhibited adverse results. Besides, western blotting revealed a concentration-dependent decrease in the level of JAK2, p-JAK2, STAT3, p-STAT3, and GSTP1 after $24 \mathrm{~h}$ of treating LoVo cells with the specific inhibitor of JAK2 (AG490). These results demonstrated that GSTP1 interacted with STAT3 without the involvement of JAK2. With regard to the previous research showing that FBX8 can degrade the expression of GSTP $1,{ }^{29}$ it was speculated that FBX8 could affect the association of GSTP1 and STAT3. Subsequent experiments confirmed that $F B X 8$ was a restraining factor for the combination of GSTP1 and STAT3.

\section{Limitations}

In this study, the analysis of the relationship between GSTP1 and STAT3 was performed using limited cohorts.
Therefore, the relationship needs to be further verified in clinical samples. Besides, we showed a region that is responsible for the interaction. However, we haven't studied this region much for further phenotypic and genetic changes.

\section{Conclusions}

In summary, GSTP1, as a downstream effector of $F B X 8$, was identified as an essential promoter and a useful prognostic marker for CRC. The GSTP1 could interact with STAT3 and upregulate the expression of STAT3 as well as of its related downstream molecules ${ }^{34}$ to promote the proliferation, invasion and metastasis of CRC. Therefore, the present study provided a potential new molecular target for the treatment of CRC metastasis. 


\section{ORCID iDs}

Feifei Wang (10) https://orcid.org/0000-0002-5962-3136 Ceng Zhang (1) https://orcid.org/0000-0002-1335-6618 Xiaohui Zhu (1) https://orcid.org/0000-0002-9031-3283 Dan Zhang @ https://orcid.org/0000-0001-5921-1898 Zhaowen Zhang (1) https://orcid.org/0000-0001-8121-5086 Shunjie Ni (1) https://orcid.org/0000-0003-1193-8574 Zhizhi Wang (1) https://orcid.org/0000-0002-1213-479X Shuyi Xu (1) https://orcid.org/0000-0002-8664-5429 Xiaoliang Lan (1) https://orcid.org/0000-0002-0582-7942 Yanqing Ding (1) https://orcid.org/0000-0003-1775-6923 Li Liang (1) https://orcid.org/0000-0001-5302-2754

\section{References}

1. Parkin DM. Global cancer statistics in the year 2000. Lancet Oncol. 2001;2(9):533-543. doi:10.1016/S1470-2045(01)00486-7

2. Steeg PS. Tumor metastasis: Mechanistic insights and clinical challenges. Nat Med. 2006;12(8):895-904. doi:10.1038/nm1469

3. Duan S, Cermak L, Pagan JK, et al. FBXO11 targets BCL6 for degradation and is inactivated in diffuse large B-cell lymphomas. Nature. 2012;481(7379):90-93. doi:10.1038/nature10688

4. Glisic B, Mihaljevic I, Popovic M, et al. Characterization of glutathione-S-transferases in zebrafish (Danio rerio). Aquat Toxicol. 2015;158: 50-62. doi:10.1016/j.aquatox.2014.10.013

5. Dong X, Sun R, Wang J, et al. Glutathione S-transferases P1-mediated interleukin- 6 in tumor-associated macrophages augments drugresistance in MCF-7 breast cancer. Biochem Pharmacol. 2020;182: 114289. doi:10.1016/j.bcp.2020.114289

6. Akbulak O, Karadag AS, Akdeniz N, et al. Evaluation of oxidative stress via protein expression of glutathione S-transferase and cytochrome p450 (CYP450) isoenzymes in psoriasis vulgaris patients treated with methotrexate. Cutan Ocul Toxicol. 2018;37(2):180-185. doi:10.1080/ 15569527.2017.1369431

7. Townsend D, Tew K. Cancer drugs, genetic variation and the glutathione-S-transferase gene family. Am J Pharmacogenomics. 2003; 3(3):157-172. doi:10.2165/00129785-200303030-00002

8. Kou X, Chen N, Feng Z, Luo L, Yin Z. GSTP1 negatively regulates Stat3 activation in epidermal growth factor signaling. Oncol Lett. 2013; 5(3):1053-1057. doi:10.3892/ol.2012.1098

9. Howells REJ, Dhar KK, Hoban PR, et al. Association between glutathione-S-transferase GSTP1 genotypes, GSTP1 over-expression, and outcome in epithelial ovarian cancer. Int J Gynecol Cancer. 2004; 14(2):242-250. doi:10.1111/j.1048-891X.2004.014207.x

10. Ruzza P, Rosato A, Rossi CR, Floreani M, Quintieri L. Glutathione transferases as targets for cancer therapy. Anticancer Agents Med Chem. 2009;9(7):763-777. doi:10.2174/187152009789056895

11. Mutallip M, Nohata N, Hanazawa T, et al. Glutathione S-transferase P1 (GSTP1) suppresses cell apoptosis and its regulation by miR-133a in head and neck squamous cell carcinoma (HNSCC). Int J Mol Med. 2011;27(3):345-352. doi:10.3892/ijmm.2010.589

12. Gao L, Fang YQ, Zhang TY, et al. GSTP1 arrests bladder cancer T24 cells in G0/G1 phase and up-regulates p21 expression. Int J Clin Exp Med. 2014;7(9):2984-2991. PMID:25356172. PMCID:PMC4211822.

13. Gurioli G, Martignano F, Salvi S, Costantini M, Gunelli R, Casadio V. GSTP1 methylation in cancer: A liquid biopsy biomarker? Clin Chem Lab Med. 2018;56(5):702-717. doi:10.1515/cclm-2017-0703

14. Buettner R, Mora LB, Jove R. Activated STAT signaling in human tumors provides novel molecular targets for therapeutic intervention. Clin Cancer Res. 2002;8(4):945-954. PMID:11948098.

15. Quintás-Cardama A, Verstovsek S. Molecular pathways: Jak/STAT pathway: Mutations, inhibitors, and resistance. Clin Cancer Res. 2013; 19(8):1933-1940. doi:10.1158/1078-0432.CCR-12-0284

16. Ma JH, Qi J, Lin SQ, et al. STAT3 targets ERR-a to promote epithelialmesenchymal transition, migration, and invasion in triple-negative breast cancer cells. Mol Cancer Res. 2019;17(11):2184-2195. doi:10.1158 /1541-7786.MCR-18-1194
17. Chen W, Dai X, Chen Y, et al. Significance of STAT3 in immune infiltration and drug response in cancer. Biomolecules. 2020;10(6):834. doi:10.3390/biom10060834

18. Chen MW, Yang ST, Chien MH, et al. The STAT3-miRNA-92-Wnt signaling pathway regulates spheroid formation and malignant progression in ovarian cancer. Cancer Res. 2017;77(8):1955-1967. doi:10.1158 /0008-5472.CAN-16-1115

19. Pan YM, Wang CG, Zhu M, et al. STAT3 signaling drives EZH2 transcriptional activation and mediates poor prognosis in gastric cancer. Mol Cancer. 2016;15(1):79. doi:10.1186/s12943-016-0561-z

20. Heichler C, Scheibe K, Schmied A, et al. STAT3 activation through IL-6/ IL-11 in cancer-associated fibroblasts promotes colorectal tumour development and correlates with poor prognosis. Gut. 2020;69(7): 1269-1282. doi:10.1136/gutjnl-2019-319200

21. Lin Q, Lai R, Chirieac LR, et al. Constitutive activation of JAK3/STAT3 in colon carcinoma tumors and cell lines: Inhibition of JAK3/STAT3 signaling induces apoptosis and cell cycle arrest of colon carcinoma cells. Am J Pathol. 2005;167(4):969-980. doi:10.1016/S0002-9440 (10)61187-X

22. Yu LZ, Wang HY, Yang SP, et al. Expression of interleukin-22/STAT3 signaling pathway in ulcerative colitis and related carcinogenesis. World J Gastroenterol. 2013;19(17):2638-2649. doi:10.3748/wjg.v19. i17.2638

23. Corvinus FM, Orth C, Moriggl R, et al. Persistent STAT3 activation in colon cancer is associated with enhanced cell proliferation and tumor growth. Neoplasia. 2005;7(6):545-555. doi:10.1593/neo.04571

24. Garcia R, Bowman TL, Niu G, et al. Constitutive activation of Stat3 by the Src and JAK tyrosine kinases participates in growth regulation of human breast carcinoma cells. Oncogene. 2001;20(20):24992513. doi:10.1038/sj.onc.1204349

25. Yu H, Pardoll D, Jove R. STATs in cancer inflammation and immunity: A leading role for STAT3. Nat Rev Cancer. 2009;9(11):798-809. doi:10.1038/nrc2734

26. Wang F, Qiao $Y, Y u$ J, et al. FBX8 acts as an invasion and metastasis suppressor and correlates with poor survival in hepatocellular carcinoma. PloS One. 2013;8(6):e65495. doi:10.1371/journal.pone.0065495

27. Wang FF, Zhang XJ, Yan YR, et al. FBX8 is a metastasis suppressor downstream of miR-223 and targeting mTOR for degradation in colorectal carcinoma. Cancer Lett. 2017;388:85-95. doi:10.1016/j. canlet.2016.11.031

28. Wu $P$, Wang $F$, Wang $Y$, et al. Significance of $F B X 8$ in progression of gastric cancer. Exp Mol Pathol. 2015;98(3):360-366. doi:10.1016/j. yexmp.2015.03.015

29. Wang FF, Xu HH, Yan YR, et al. FBX8 degrades GSTP1 through ubiquitination to suppress colorectal cancer progression. Cell Death Dis. 2019;10:351. doi:10.1038/s41419-019-1588-z

30. Robinson RL, Sharma A, Bai S, et al. Comparative STAT3-regulated gene expression profile in renal cell carcinoma subtypes. Front Oncol. 2019;9:72. doi:10.3389/fonc.2019.00072

31. Masuda M, Suzui M, Yasumatu R, et al. Constitutive activation of signal transducers and activators of transcription 3 correlates with cyclin D1 overexpression and may provide a novel prognostic marker in head and neck squamous cell carcinoma. Cancer Res. 2002;62(12): 3351-3355. PMID:12067972.

32. Kawada M, Seno H, Uenoyama $Y$, et al. Signal transducers and activators of transcription 3 activation is involved in nuclear accumulation of beta-catenin in colorectal cancer. Cancer Res. 2006;66(6): 2913-2917. doi:10.1158/0008-5472.CAN-05-3460

33. Morikawa T, Baba Y, Yamauchi M, et al. STAT3 expression, molecular features, inflammation patterns, and prognosis in a database of 724 colorectal cancers. Clin Cancer Res. 2011;17(6):1452-1462. doi:10.1158 /1078-0432.CCR-10-2694

34. Buchert M, Burns $C J$, Ernst M. Targeting JAK kinase in solid tumors: Emerging opportunities and challenges. Oncogene. 2016;35(8): 939-951. doi:10.1038/onc.2015.150 